\title{
G

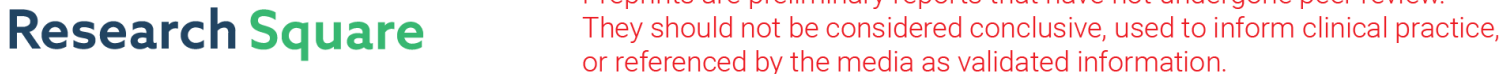

\section{Validation of a QTL for grain size and weight using an introgression line from a cross between Oryza sativa and Oryza minuta}

\section{Yue Feng}

China National Rice Research Institute

Xiaoping Yuan

China National Rice Research Institute

\section{Yiping Wang}

China National Rice Research Institute

\section{Yaolong Yang}

China National Rice Research Institute

\section{Mengchen Zhang}

China National Rice Research Institute

Hanyong Yu

China National Rice Research Institute

\section{Qun Xu}

China National Rice Research Institute

\section{Shan Wang}

China National Rice Research Institute

Xiaojun Niu

China National Rice Research Institute

Xinghua Wei ( $\nabla$ weixinghua@caas.cn) https://orcid.org/0000-0002-0883-3694

\section{Original article}

Keywords: Oryza sativa, Oryza minuta, Introgressive line, Grain size and weight, Quantitative trait loci

Posted Date: January 4th, 2021

DOI: https://doi.org/10.21203/rs.3.rs-54218/v2

License: (1) This work is licensed under a Creative Commons Attribution 4.0 International License. Read Full License 
Version of Record: A version of this preprint was published at Rice on May 20th, 2021. See the published version at https://doi.org/10.1186/s12284-021-00472-1. 


\section{Abstract}

Background: Grain size and weight are important target traits determining grain yield and quality in rice. Wild rice species possess substantial elite genes that can be served as an important resource for genetic improvement of rice. In this study, we identify and validate a novel QTL on chromosome 7 affecting the grain size and weight using introgression lines from cross of Oryza sativa and Oryza minuta.

Results: An introgression line 'IL188' has been achieved from a wild species Oryza minuta ( $2 n=48, B B C C$, W303) into 0 . sativa japonica Nipponbare. The F2 and F2:3 populations derived from a cross between IL188 and Nipponbare were used to map QTLs for five grain size traits, including grain length (GL), grain width (GW), grain length to width ratio (LWR), grain thickness (GT) and thousand grain weight (TGW). A total of 12 QTLs for the five grain traits were identified on chromosomes $1,2,3,6,7$, and 8. The QTL-qGL7 controlling GL on chromosome 7 was detected stably in the F2 and F2:3 populations, and explained $15.09-16.30 \%$ of the phenotypic variance. To validate the effect of $q G L 7$, eight residual heterozygous line (RHL) populations were developed through selfing four F2:3 and four F2:4 plants with different heterozygous segments for the target region. By further developing SSR and Indel markers in the target interval, $q G L 7$ was delimited to a $261 \mathrm{~kb}$ region between Indel marker Y7-12 and SSR marker Y7-38, which also showed significant effects on grain width and thousand grain weight. Comparing with the reference genome of Nipponbare, stop or frameshift mutations in the exon of the three putative genes LOC_Os07g36830, LOC_Os07g36900 and LOC_Os07g36910 encoding F-box domain-containing proteins may be the candidate genes for $q G L 7$. Scanning electron microscopy analysis of the glume's epidermal cells showed that the cell length and width of NIL- $q G L$ ZL188 was higher than NIL$q G L \mathbb{N}$ ip, indicating that $q G L 7$ increases grain size and weight by regulating cell expansion.

Conclusions: In this study, we detected 12 QTLs regulating grain size and weight using an introgression line from a cross between an Oryza sativa and Oryza minuta. Of these loci, we confirmed and delimited the $q G L 7$ to a $\sim 261 \mathrm{~kb}$ region. Three putative genes, LOC_Os07g36830, LOC_Os07g36900 and LOC_Os07g36910 encoding F-box domain-containing proteins may be the candidate genes for $q G L 7$. These results provide a basis for map-based cloning of the $q G L 7$ gene and useful information for marker assisted selection in rice grain quality improvement.

\section{Background}

Rice (Oryza sativa L.) is one of the most important cereal crops in Asiaand is the main staple food for the majority of peoples in the world.Breeding ofhigh-yielding rice is crucial for meeting the fooddemand of theincreasing world population (Ikeda et al. 2013). Grain yield in rice isdetermined by three major components: the number ofpanicles, the number of grains per panicle, and grainweight(Huang et al. 2013).Among these, themost reliable trait is grain weight, which is largely determined by grain size, which is specified by its three dimensions (length, width, and thickness) andthe degree of filling(Xiong and Zhang 2010).

Grain size and weight are important components determining ricegrain yield, and they are controlled by multiple quantitative trait loci (QTLs) (Zhang et al. 2012; Yu et al. 2018). To date, over 400 QTLs 
modulating grain size and weight have been identified and are distributed on each of rice's 12 chromosomes (Huang et al. 2013; Zuo and Li 2014; Kashifet al. 2020). However, only a few major QTLs including GS3, qSW5,GW2, qGL3/GL3.1,GW8, GL7/GW7, TGW6and GS9 have been isolated by map-based cloning methods (Mao et al. 2010; Shomura et al. 2008; Song et al. 2007; Qi et al. 2012; Zhang et al. 2012; Wang et al. 2012; Wang et al. 2015a; Wang et al. 2015b; Ishimaru et al. 2013; Zhao et al. 2018).The isolation of these genes has enhanced ourknowledge of the molecular regulatory mechanisms responsible for grain size and weight (Song et al. 2008).

Oryza minuta $(2 n=48, B B C C)$ is an allotetraploidwild species, which is endemic to Philippines and Papua New Guinea. This species belongs to the Oryza officinalis complex and harbors useful genes for resistance to blast blight, bacterial blight, brown planthopper and sheath blight (Amante et al. 1992; Brar and Khush 1997). However, low crossability and limited recombination between unrelated genomes limit the transfer QTLs from Oryza minuta to cultivars. Following the availability of advancedbackcross quantitative trait loci (AB-QTL) approach proposed byTanksley and Nelson (1996),several studies have been reported to identifythe QTLscontrollingyieldand quality-related traitsand to simultaneously transfer them from wild to cultivated species(Xiao et al. 1998; Thomson et al. 2003; Yoon et al. 2006; Tian et al. 2006; MallikarjunaSwamy et al. 2012; Yun et al. 2016). However, few attempts have been made to identify and capture yield-related QTLs from Oryza minuta into cultivars.

In the present study, we used an advanced across line IL188 from a cross between the japonica variety, Nipponbare, Oryza sativa and a wild accession, W303, Oryza minuta, as the donor parent, to map QTLs for rice grain size traits. The objectives of this study were: (1) to reliably identify novel genomic regions associated with grain size traits from W303 (Oryza minuta), (2) to evaluate the effects of introgressive segments on grain size traits, (3) to fine mapping the QTL- $q G L 7$ and validate the effects of $q G L 7$ for rice grain size and weight on chromosome 7.

\section{Results}

\section{Genetic background of IL188}

A total of 512 SSR markers were screened for polymorphism between W303 and Nipponbare. Among them, 185 markers produced polymorphic bands between the parents. These 185 polymorphic markers were further used to assay the genotype of IL188. Thirty of these markers (16.2\%) showed W303 genotype, which covered 11 regions distributing on six chromosomes. The introgressed segments distributed on chromosomes 1, 2(two), 3, 5, 6 (two), 7 (two), and 8 (two), respectively (Fig. 3). These30 markers were further used to genotype the F2 andF2:3 populations derived from a cross between Nipponbare and IL188.

\section{Trait performance ofthe parents, $\mathrm{F} 2$ and $\mathrm{F} 2: 3$ populations}

The phenotypic values of the two parents of fiveagronomic traits, including GL, GW, GT, LWR, and TGW were shown in Table 1. Compared with Nipponbare, IL188 had higher values for GL, GW, LWR, and TGW but lower values for GT. The frequency distributions of the five grain sizetraits in the F2 and F2:3 populationsdisplayed a continuous variation(Fig. 4). All these traits expect TGW showed two-way 
transgressive segregation and followed a near normal distribution in the both populations. The results fulfill the requirement of QTL mapping.

\section{Correlation analysis of five grain traits}

The correlation coefficients among the five grain traitsin the F2 and F2:3 populationswere shown in Table 2. The correlation coefficients ranged from -0.677 to 0.614 and -0.662 to 0.598 , respectively, in the F2 and F2:3 populations.Significant correlation was observed for each pair-wise combination except that between GL and GT, LWR and TGW. In the both populations, GL showed positive correlation with GW, LWR and TGW, GW displayed strong positive correlation with GT and TGW, while LWR showed negative correlation with GW and GT.

\section{QTLs for grain size traits in the F2 and F2:3populations}

A total of 12 QTLs for five grain sizetraits were detected on chromosomes 1, 2, 3, 6, 7, and 8 in the F2 and F2:3 populations (Table 3). The phenotypic variance explained by each QTL ranged from $4.72 \%$ to $16.30 \%$.Four of these regions were found to affect two traits. The RM7341-RM128interval on the chromosome 1 and RM12924-RM5812 interval on chromosome 2 showed consistent effects on GL and LWR in both populations. In theRM7341-RM128interval, $q G L 1$ and $q L W R 1$ explained phenotypic variances by $8.77 \%$ and $7.55 \%$ in the F2 population, and $9.13 \%$ and $7.40 \%$ in the $\mathrm{F} 2: 3$ population, respectively. In the RM12924-RM5812 interval, qGL2 and qLWR2explained phenotypic variances by $6.90 \%$ and $5.02 \%$ in the F2 population, and $7.09 \%$ and $5.68 \%$ in the $\mathrm{F} 2: 3$ population, respectively. The enhancing alleles of these QTLs all derived from IL188. The RM500-RM429 interval on chromosome 7 showed consistent effects on GL and TGW in the both populations. The qGL 7 and qTGW7 explained phenotypic variances by $16.30 \%$ and $9.97 \%$ in the F2 population, and $15.09 \%$ and $6.65 \%$ in the $\mathrm{F} 2: 3$ population, respectively. The enhancing alleles of the two QTLs also derived from IL188. The RM3845-RM6948 on chromosome 8 exhibitedsignificant effects on GT and LWR only in the F2:3 population. The $q G T 8$ and $q L W R 8$ explained $7.41 \%$ and $4.72 \%$ of phenotypic variances, with enhancing alleles derived from Nipponbare and IL188, respectively. The other four regions, which covered RM6307-RM5807, RM3199-RM3684, RM7158-RM276, RM408-RM3702 on chromosome 2, 3,6 and 8, respectively, were each detected for a single trait, with $R 2$ ranging from $4.85 \%$ to $7.06 \%$.

Among these regions, the RM500-RM429 interval on chromosome 7 showedthe largest effectfor GL and relatively stable QTLs for TGW. Therefore, the region was chosen for further validation. For ease of description, the $q G L 7$ and $q T G W 7$ detected in this region were integrated as $q G L 7$.

\section{Substitution mapping of $q G L 7$ and sequence analysis of candidate genes}

Four NIL-F2 populations carrying heterozygous segments overlapped inthe RM500-RM429 interval wereconstructed,including R1, R2, R3 and R4.Significant genotypic effects were detected for the three grain size traits in R2 and R3. In the two populations, the additive effects were 0.115 and 0.109 for GL, 0.065 and 0.050 for GW,0.621 and 0.907 for TGW,explained phenotypic variances by $19.54 \%$ and $15.46 \%, 21.65 \%$ and $10.24 \%$,and $16.09 \%$ and $16.86 \%$ (Table 4 ). The enhancing allele was derived from IL188, the same as what was found in the F2 and F2:3 populations. The additive effects and $R 2$ were similar between R2 and R3, indicated that $q G L 7$ located in the common segregating regions of the two populations. In 
R1 and R4, no significant effect was detected for any traits, indicated that $q G L 7$ located outside of segregating regions of the two populations. As shown in Fig. 5, this is an interval flanked bymarkers Y7-3 and Y7-4, correspondingto a 725-kb region in the Nipponbare genome (Fig. 5).

Following the update target regions, other four NIL-F2 populations were developed, including R5, R6, R7 and R8.Significant genotypic effects were detected in R6 and R8, but not in R5 and R7. InR6 and R8, the additive effects were 0.129 and 0.074 for $\mathrm{GL}, 0.026$ and 0.031 for $\mathrm{GW}, 0.595$ and 0.494 for TGW,explained phenotypic variances by $48.52 \%$ and $18.12 \%, 16.44 \%$ and $12.77 \%$,and $25.38 \%$ and $9.92 \%$ (Table 4 ). Again, the enhancing allele was derived from IL188. These results indicated that $q G L 7$ was located within the common segregating regions of $\mathrm{R} 6$ and $\mathrm{R} 8$ but outside the segregating region of $\mathrm{R} 5$ and $\mathrm{R} 7$. Consequently, $q G L 7$ was delimited into a261-kb region flanked byY7-12 and Y7-38 (Fig. 5).

In the 261-kb genomic region of the Nipponbare genome, a total of thirty-seven putative genes were predicted based on the Nipponbare sequence (Os-Nipponbare-Reference-IRGSP-1.0). Whole-genome resequencing was performed on the NIL-qGL $\mathrm{JL188}$. Compared with the Nipponbare reference genome, seven stop code mutations and ten frameshift mutations were identified in the exonic region of the twelve genes (Table S3). One of them, LOC_Os07g36850 encodes a putative transposon protein, four genes encode retrotransposon proteins and four genes encode expressed protein of unknown function. In addition, LOC_Os07g36900 encodes a protein containing F-box and LRR motifs, both LOC_Os07g36830 and LOC_Os07g36910 encodeF-box proteins.

\section{Histocytological Analysis}

The homozygous plants were selected from R6 and R8. They were selfed to develop two NIL populations. The effect of $q G L 7$ was further validated using these two populations.Compared with NIL- $q G L \mathbb{N}$ Nip, the GL and GW in the NIL-qGL $7 L 188$ were significantly larger (Fig. 6A-C), thus resulting in a larger TGW (Fig. 6D). These indicated that $q G L 7$ had stable effects on grain size traits.

In addition, we examined the cell length and width ofepidermal cells of the outer and inner spikelet hulls of NIL-qGL 7 Nip and NIL-qGL 7 L188by scanning electron microscopy. Both the length and width of epidermal cells ofthe outer and inner spikelet hullswere increased in NIL- $q G L$ ZL 188compared with those in NIL-qGL Nip (Fig. 6E-L).Additionally, we investigated the cell number in the vertical and lateral direction of the outer spikelet hulls of the NIL-qGL Nip and NIL-qGL ZL188 by scanning electron microscopy. No significant difference in total cell number in the longitudinal and lateral direction of spikelet hulls was observed between NIL- $q$ GL Nip and NIL- $q G L$ ZL188 (Figure S1B-C). These results indicate that $q G L$ 7regulates grain size bypromoting cell expansion.

\section{Discussion}

Common wild rice is the wild ancestor ofcultivated rice (Second, 1982; Oka, 1988; Wang et al. 1992). As the ancestor of cultivated rice, wild rice has beenwell recognized as an extremely important resourcefor rice improvement, since it carries many beneficialagronomic traits which have been lost in the cultivatedrice through natural and human selection (Sun et al. 2001; Sakai andltoh 2010).In the present study, an advanced backcross line, IL188, was developed from a cross between Nipponbare and O. minuta. The F2 and 
F2:3 populations derived from a cross between Nipponbare and IL188 was used to identify the QTLs controlling grain size and grain weight. A total of 12 putative QTLs for grain size and grain weight were detected in the F2 and F2:3 populations, and 9 of which were commonly detected in both populations.

A comparison of the QTL regionsfrom this study with those seen in previous rice linkagemaps (http://www.gramene.org) revealed that six regionswere sharedacross studies.For GL and LWR, one QTL was detected in the interval RM7341-RM128 on chromosome 1. Wan et al. (2005) also detected a stable QTL for the same traits in the similar regions on chromosome 1, andQi et al. (2017) detected a QTL for LWR closely linked with the marker RM128. One locus associated with GL and LWR ( $q G L 2$, and qLWR2) were located in the interval RM12924-RM5812 on chromosome 2. Interestingly, Yoon et al. (2006) confirmed a locus associated with TGW, GW, GT, and LWR in the same region using an advanced backcross population between 0 . Grandiglumis and $O$. sativa. Two QTLs for GW and GT wererespectively located in the vicinity of QTLs detected in previous studies (Yoon et al. 2006; MallikarjunaSwamy et al. 2012; Qi et al. 2017). In our study, one QTL was detected for TGW in the interval RM6307-RM5807 on chromosome 2, and Xue et al. (2019) also detecteda QTL for TGW in the nearby region.

More importantly, one major QTL, qGL7, was detected for GL and TGW in the interval RM500-RM429 on chromosome 7 in the $\mathrm{F} 2$ and $\mathrm{F} 2: 3$ populations. The 0 . minuta introgressive line alleles could increase $\mathrm{GL}$ and TGW. The qGL 7 could explain 15.09-16.30\% and 6.65-9.97\% of the phenotypic variation for GL and TGW, respectively. The 0 . minuta allele at locus $q G L 7$ increased GL and TGW by an average of $0.19 \mathrm{~mm}$ and $0.65 \mathrm{~g}$, respectively. Interestingly, Rahman et al. (2007) also detected a QTL for GL and TGW in the same region using an F2:3population between 0 . minuta introgression line and $O$. sativa. This result indicated that there really exist a stable QTL controlling grain size and grain weight, and the 0 . minuta allele could positively regulate grain size and grain weight. As we known, some major-effect QTLs for grain size and weight on chromosome 7 have been fine mapped and cloned in previous studies. Bai et al. (2010) detected a pleiotropic QTL for grain size and this QTL $q G L 7$ was narrowed down to within a 258-kb region.Shao et al. and Qiu et al. identified a major QTL GS7/ qSS7 on the long arm of chromosome 7 for grain size. Subsequently, This GL7/ GW7 (the same as GS7/ qSS7) gene has been cloned by Wang et al. (2015a) and Wang et al. (2015b). They have found that copy number variations at GL7/GW7 locus cause elevated expression of GL7 and thus an increase in grain length. The grain size gene GLW7, encoding the plant-specific transcription factorOsSPL13, has been isolated and functionally characterized using GWAS approach (Si et al. 2016). Xu et al. (2015) identified a dominant big grain mutant BG2 that encoded a cytochrome P450, OsCYP78A13on chromosome 7. Here, we have defined the locus $q G L 7$ to a $261 \mathrm{~kb}$ region on the long arm of chromosome 7. By comparing the physical location of $q G L$ 7with the reported grain size QTLs on chromosome 7, we found that $q G L 7$ is a novel QTL for regulating rice grain size.

Compared with the Nipponbare reference genome, seven stop code mutations and ten frameshift mutations were identified in the exonic region of the twelve genes of NIL- $q G L$ ZL188in the 261-kb region. Three putative genes of them, LOC_Os07g36830, LOC_Os07g36900 and LOC_Os07g36910 encoded F-box domain-containing proteins. F-box proteins are the substrate-recognition components of SCF (SKP1-Cul1-F-box) type E3 ubiquitin protein ligases (Skowyra et al. 1997; Feldman et al. 1997), which participate in the regulation of many physiological processes and play a key role in cell division, signal transduction, development and metabolism 
(Patton et al. 1998). In rice, GW2 encodes a RING-type protein with E3 ubiquitin ligase activity, which is known to function in the degradation by the ubiquitin-proteasome pathway (Song et al. 2007). In addition, Chen et al. 2013 reported that overexpression of OsFBK12 (encoding an F-box protein) could increase grain size in rice. Therefore, we suppose that the three putative genes, LOC_Os07g36830, LOC_Os07g36900 and LOC_Os07g36910 encoded F-box domain-containing proteins may be the candidate genes for $q G L 7$. In future, transgenic studies will be carried out for the three Fbox domain-containing genes identified in the $q G L 7$ locus to further elucidate the molecular mechanism of $q G L 7$ involving in regulation of rice grain size.

Classical quantitative genetics assumes that traitcorrelations are the result of either pleiotropic effects orthe tight linkage of genes (Wan et al. 2005).In this study, $q G L 1 / q L W R 1$ and $q G L 2 / q L W R 2$ were mapped in the same interval on chromosome 1 and chromosome 2, respectively, and the positive alleles were all derived from 0 . minuta. As well, $q G L 7$ and $q T G W 7$ shared the same confidence interval on chromosome 7 and their effect acted in the same direction.Co-localizationof these QTLs, as the result of either pleiotropic effectsor close linkage, could provide an explanation for thegenetic basis of high trait correlations, which ranged from 0.488 between GL and LWR to 0.614 between GL and TGW.

Transfer and utilization useful genes from wild rice into cultivated varieties are effectiveand aim to improve grain yield, quality, and crop genetic diversity (Brar and Khush 1997; Xie et al. 2008; Yun et al. 2016; Qi et al. 2017).However, efforts to improve rice grain traits of modern cultivars using O. minuta as donor parents are limited. In the present study, 0 . minuta alleles increase rice grain traits in the Nipponbare background at most QTLs, revealing the possibility that $O$. minuta alleles could improve grain traits. Although a number of genes/QTLs involvedin the regulationof grainsizehave been cloned in rice,the molecular mechanisms of how grain size isregulated remain unknown.In this study, we found that $q G L 7$ could increase both grain length and grain weight, and the isolationof $q G L 7$ will be beneficialin better understanding of the regulation mechanism of grain size in rice. In addition, our continuous work will be helpful in improvingriceyield and quality bymolecular design breeding.

\section{Conclusions}

An introgression line IL188 was identified, which exhibited increased grain size and weight. A total of 12 QTLs for five grain traits were detected using F2 and F2:3populations derived from crosses between IL188 and Nipponbare. One of the QTLs, qGL7was delimited to a 261 kb region on the long arm of chromosome 7,and three putative genes, LOC_Os07g36830, LOC_Os07g36900 and LOC_Os07g36910encoding F-box domain-containing proteins may be the candidate genes for qGL7. TheqGL7 increases grain size and weight by regulating cell expansion. These results will be helpful not only for understanding the genetic basis of grain size traits, but also simultaneously improvinggrain size and weightthrough marker-assisted selection (MAS) in rice breeding programs.

\section{Materials And Methods}

\section{Plant materials}


The introgression line, IL188, derived from an interspecific cross between Oryza sativajaponica Nipponbare and a wild species Oryza minuta W303 collected from the Germplasm Resource Center of IRRI, followed by three backcrosses with Nipponbare and aided by embryo rescue and subsequently self-pollinated for four generations. IL188 showed significantly longer grain length and higher grain weight than the recurrent parent Nipponbare (Fig. 1). To elucidate the genetic basis of the grain size and weight variation, an F2 population consisting of 166 individuals was constructed by selfing the F1 between the female parent IL188 and male parent Nipponbare,and the F2:3population was derived from the selfed seeds of the F2plants.

Following the initial outcome of QTL analysis, four residual heterozygous plants were selected from the F2:3 populations, carrying sequential heterozygous segments covering the interval RM500-RM429. They were selfed, and four NIL-F2populations were constructed.They contained 180, 184, 184and195 plants and were named as R1, R2, R3 and R4, which carried overlapping heterozygous segments in the interval RM11RM1135, RM11-Y7-2, RM11-Y7-2 and RM11-Y7-4, respectively (Fig. 2). The genomic background of the R1, R2, R3 and R4 populations was shown in Table S1. The R1, R2, R3 and R4 populations which was respectively only heterozygous in the target interval and basically homozygous in the genomic background were used for the substitution mapping of $q G L 7$.

Four other plants were further selected from the R3 population carrying sequential heterozygous segments covering the interval Y7-3-Y7-4. They were selfed, and four NIL-F2populations were constructed. They contained 130, 144, 146and140 plants and were named as R5, R6, R7 and R8, which carried overlapping heterozygous segments in the interval Y7-4-RM21787, Y7-4-RM455, RM21787-Y7-12 and RM21787-Y7-13, respectively (Fig. 2). The R5, R6, R7 and R8 populations were used for further substitution mapping of $q G L 7$.Non-recombinant homozygous plants were further identified in the R6 and R8 populations and selfed. Two sets ofNILs were developed, each consisting of 20IL188 homozygous lines and 20 Nipponbare homozygous lines.

The F2 and F2:3populations were grown at the Hangzhou Experiment Station of China Rice Research Institute (CNRRI), Zhejiang (N 30 $32^{\prime}$, E $\left.120^{\circ} 12^{\prime}\right)$, China, and the Lingshui Experiment Station of CNRRI, Hainan (N $\left.18^{\circ} 48^{\prime}, \mathrm{E} 110^{\circ} 02^{\prime}\right)$, China, in the summer and winter of 2014. The NIL-F2populations and two sets ofNILswere planted at the Hangzhou Experiment Station of CNRRI in the summer of 2015, 2016 and 2017. The F2and NILF2populations were planted with $20 \mathrm{~cm}$ between plants and $30 \mathrm{~cm}$ between rows. The F2:3 families and two sets of NILswere grown in a randomized complete block design with two replications, five rows per plot, 8 plants per row, $20 \mathrm{~cm}$ between plants within each row and $30 \mathrm{~cm}$ between rows. The field management followed the standard agronomic practices.

\section{Grain size trait evaluation}

For the F2and NIL-F2populations, the plants were individually harvested for traitevaluation.For theF2:3 population andNILs-qGL Nip and NILs- $q$ GL ZL188, ten plants in each line wereharvested in bulk for traitevaluation. Five grain size traits were evaluated in each population. For grain length (GL), grain width (GW) and grain thickness (GT), 20 full-filled rice grains were randomly selected and individually measuredusing an electronic digital display vernier caliper. The averaged values of the20 grains were used fordata analysis. The 
grain length-width ratio (LWR) isequal to GL divided by its GW.Thousand grain weight (TGW) was evaluated by measuring theweight of 200 randomly selected full-filled grains per F2 plant.The phenotypic evaluations of F2:3 family, NIL-F2populationand NILlines were the same as those for F2 plants describedabove.

\section{Scanning Electron Microscopy}

The spikelets of NIL-qGLTNip and NIL-qGL JL188 were collected at maturity stage. The samples were fixed in FAA solution (formalin:glacial acetic acid: ethanol in 1:1:18 ratio by volume) at $4{ }^{\circ} \mathrm{C}$ for $24 \mathrm{~h}$, then dehydrated by a graded ethanol series, and were dried by critical-point drying method. The samples were observed under the scanning electron microscope (HITACHI, S-3000N). The spikelet epidermal cell sizewas measuredusing image $J$ software.

\section{DNA extraction and molecular marker analysis}

DNA was extracted from fresh leaves samples following the CTAB method (Murray and Thompson 1980) with minor modifications. A total of 512 SSR markers with good genome coverage were selected to detect the polymorphisms between parents W303 and Nipponbare, 185 of which distributed across all 12 chromosomes showed polymorphisms between the two parents. Furthermore, 30 polymorphic SSR markers between IL188 and Nipponbare were used to genotype the F2 and F2:3 populations. Sixteen markers were used for finemapping (Table S2).

\section{Linkage map construction and data analysis}

A genetic linkage map was constructed using MAPMAKER/EXP version 3.0 (Lander et al. 1987). The Kosambi mapping function (Kosambi, 1944) was used to transform the recombination frequency into cM. Composite interval mapping (CIM) was carried out to scan the introgressive genomic regions for putative QTLs using Windows QTL Cartographer 2.5 (http:// statgen.ncsu.edu/qtlcart/WQTLCart.htm). The LOD threshold of 2.5 was usedfor declaring the presence of a putative QTL ina givengenomic region.Nomenclature of QTLswas conducted as described by McCouch et al. (1997).

Phenotypic differences between IL188 and Nipponbareand between two homozygous lines in the NIL populations were compared using the student's test. Correlation analysis of grain size traits were performed using SPSS software.

\section{Additional Files}

\section{Supplementary information}

Supplementary Table S1: The genomic background of the R1-R4 populations.

Supplementary Table S2: Primers used for fine mapping.

Supplementary Table S3: The variations of NIL- $q G L 7 L 188$ in the 261-kb region compared with the Nipponbare reference genome. 
Supplementary Figure S1: Comparison of grain size and cell number in the outer spikelet hulls along the vertical and lateral direction between NIL-qGL Nip and NIL-qGL ZL188. Scale bar, $1 \mathrm{~mm}$. A, Mature grains of NIL-qGL $7 L 188$ (left) and NIL-qGL Nip (right). B-C, The cell number in the longitudinal and lateral direction of outer spikelet hulls.

\section{Declarations}

\section{Acknowledgments}

We wish to thank Dr. Zhang Zhenhua for careful corrections and valuable suggestions on the revision.

\section{Authors' Contributions}

YF designed the experiments; $X Y, Y W, Y Y, M Z, H Y, Q X, S W$, and $X N$ performed experiments and analyzed the data; $Y F$ and $X W$ wrote the manuscript. All authors read and approved the final version of the manuscript.

\section{Funding}

This study was supported by the Major Scientific and Technological Project forNew Varieties Breeding of Zhejing Province (2016C02050-6-1),Zhejiang Provincial Natural Science Foundation of China (LY15C130005).

\section{Availability of Data and Materials}

The datasets supporting the conclusions of this article are included within the article.

\section{Consent for Publication}

All authors are consent for publication.

\section{Competing interests}

The authors declare that they have no competing interests.

\section{Abbreviations}

QTL: Quantitative trait locus; IL: Introgression line; RHL: Residual heterozygous line; SSR: Simple sequence repeat; GWAS: Genome wide association study; MAS: Molecular assisted selection; NIL: Near isogenic line

\section{References}

Amante-Bordeos A, Sitch LA, Nelson R, Dalmacio RD, Oliva NP, Aswidinnoor H, Leung H(1992) Transfer of bacterial blight and blast resistance from the tetraploid wild rice Oryza minutato cultivated rice, Oryza sativa. Theor Appl Genet84:345-354 
Bai XF, Luo LJ, Yan WH, Kovi MR, Zhan W, Xing YZ (2010)Genetic dissection of rice grain shape using arecombinant inbred line population derived fromtwo contrasting parents and fine mapping apleiotropic quantitative trait locus qGL7. BMC genet 11:16

Brar DS, Khush GS (1997)Alien introgression in rice. Plant Mol Biol35:35-47

Chen Y, Xu YY, Luo W, Li WX, Chen N, Zhang DJ, Chong K (2013) The F-box protein OsFBK12 targets OsSAMS1 for degradation and affects pleiotropic phenotypes, including leaf senescence in rice. Plant Physiol 163:16731685

Feldman RM, Correll CC, Kaplan KB, Deshaies RJ (1997) A complex of Cdc4p, Skp1p, and Cdc53p/cullin catalyzes ubiquitination of the phosphorylated CDK inhibitor Sic1p. Cell 91:221-230

Huang R, Jiang L, Zheng J, Wang T, Wang H, Huang Y, Hong Z (2013)Genetic bases of rice grain shape: somany genes, so little known. Trends Plant Sci 18:218-226

Ikeda M, Miura K, Aya K, Kitano H, Matsuoka M (2013)Genes offering the potential for designing yield-related traitsin rice. CurrOpin Plant Biol16:213-220

Huang RY, Jiang LR, Zheng JS, Wang TS, Wang HC, Huang YM, Hong ZJ (2013)Genetic bases of rice grain shape: somany genes, so little known. Trends Plant Sci18:218-226

Ishimaru K, Hirotsu N, Madoka Y, Murakami N, Hara N, Onodera H, Kashiwagi T, Ujiie K, Shimizu B, Onishi A, Miyagawa $\mathrm{H}$, Katoh E (2013)Loss of function of the IAA-glucose hydrolase gene TGW6enhances rice grain weight and increases yield. NatGenet 45(6):707-711

Kashif H, Zhang YX, Workie A, Aamir R, Adil A, Md. Hasanuzzaman R, Wang H, Shen XH, Cao LY, Cheng $\mathrm{SH}$ (2020)Association mapping of quantitative trait loci for grain size in introgression line derived from oryzarufipogon. Rice Sci27(3):246-254

Kosambi, DD (1944) The estimation of map distance from recombination values. Ann Eugen 12:172-175.

Lander ES, Green P, Abbrahamson J, Barlow A, Daly MJ, Lincoln SE, Newburg L (1987) MAPMAKER: an interactive computer package for constructing primary genetic linkage maps of experimental and natural populations. Genomics 1:174-181

MallikarjunaSwamy BPM, Kaladhar K, Shobha Rain N, Prasad GSV, Viraktamath BC, Ashok Reddy G, Sarla N (2012)QTL analysis for grain quality traits in2 BC2F2populations derived from crosses between Oryza sativa cv Swarnaand 2 accessions of O. nivara. J Hered 103(3):442-452

Mao HL, Sun SY, Yao JL, Wang CR, Yu SB, Xu CG, Li XH,Zhang QF (2010) Linking differential domain functions of theGS3 protein to natural variation of grain size in rice. Proc NatIAcad Sci USA 107:19579-19584

McCouch SR, Cho YG, Yano M, Paul E, Blinstrub M, Morishima H, Kinoshita T (1997) Report on QTL nomenclature. Rice Genet Newsl14:11-13 
Murray MG, Thompson WF (1980) Rapid isolation of high molecular weight plant DNA. Nucleic Acids Res 8:4321-4325

Oka HI (1988) Origin of cultivated rice. Developments in Crop Science. Vol. 14, Japan Scientific Society Press okyo and Elsevier. Amsterdam.

Patton EE, Willerns AR, Tyers M (1998) Combinatorial control in ubiquitin-dependent proteolysis: don't Skp the F-box hypothesis. Trends Genet 14:236-243

Qi L, Sun Y, Li J, Sun L, Zheng XM, Wang X, Li K, Yang Q, Qiao W (2017)Identify QTLs for grain size and weight in common wild rice using chromosomesegment substitution lines across six environments. Breeding Sci $67: 472-482$

Qi P, Lin YS, Song XJ, Shen JB, Huang W, Shan JX, Zhu MZ,Jiang LW, Gao JP, Lin HX (2012) The novel quantitative traitlocus GL3.1 controls rice grain size and yield by regulatingCyclin-T1;3. Cell Res 22(12):16661680

Qiu XJ, Gong R, Tan YB, Yu SB (2012)Mapping and characterization of the major quantitative traitlocus qSS7 associated with increased length and decreased widthof rice seeds. Theor Appl Genet 125(8):1717-1726

Rahman ML, Chu SH, ChoiM, Qiao YL, Jiang WZ, Piao R, Khanam S, Cho Y, Jeung J, Jena KK, Koh $\mathrm{H}$ (2007) Identification of QTLs for some agronomic traits in rice usingan introgression line from Oryza minuta. Mol Cells 24(1): 16-26

Sakai H, Itoh T (2010) Massive gene losses in Asian cultivated rice unveiled by comparative genome analysis. BMC Genom 11:121

Second G (1982)Origin of the genic diversity of cultivated rice (Oryzaspp.): study of the polymorphism scored at 40 isozyme loci.(Jpn J Genet) 57:25-57

Shao GN, Wei XJ, Chen ML, Tang SQ, Luo J, Jiao GA, Xie LH, Hu PS (2012)Allelic variation for a candidate gene for GS7, responsiblefor grain shape in rice. Theor Appl Genet 125(6):1303-1312

Shomura A, Izawa T, Ebana K, Ebitani T, Kanegae H, Konishi S,Yano M (2008) Deletion in a gene associated with grain sizeincreased yields during rice domestication. Nat Genet 40(8):1023-1028

Si LZ, Chen JY, Huang XH, Gong H, Luo JH, Hou QQ, Zhou TY, Lu TT, Zhu JJ, Shangguan YY, Chen EW, Gong CX, Zhao Q, Jing YF, Zhao Y, Li Y, Cui LL, Fan DL, Lu YQ, Weng QJ, Wang YC, Zhan QL, Liu KY, Wei XH, An K, An G, Han B (2016) OsSPL 13 controls grain size in cultivated rice. Nat Genet 48:447-456

Skowyra, D, Craig KL, Tyers M, Elledge SJ, Harper JW (1997) F-box proteins are receptors that recruit phosphorylated substrates to the SCF ubiquitin-ligase complex. Cell 91:209-219

Song XJ, Ashikari M (2008) Toward an optimum return from crop plants. Rice 1:135-143 
Song XJ, Huang W, Shi M, Zhu MZ, Lin HX (2007)A QTL for rice grain width and weight encodes a previously unknown RING-type E3 ubiquitin ligase. Nat Genet 39:623-630

Sun CQ, Wang XK, Li ZC, Yoshimura A, Iwata N (2001)Comparison of the genetic diversity of common wild rice (Oryza rufipogon Griff.) and cultivated rice (O. sativa L.) using RFLP markers. Theor Appl Genet 102:157-162

Swamy BPM, Kaladhar K, Rani NS, Prasad GSV, Viraktamath BC, Reddy GA, Sarla N (2012)QTL analysis for grain quality traits in2 BC2F2populations derived from crosses between Oryzasativa cv Swarnaand 2 accessions of $O$. nivara. J Hered 103(3):442-452

Tanksley SD, Nelson JC (1996) Advanced backcross QTL analysis: a method for the simultaneous discovery and transfer of valuable QTLs from unadapted germplasm into elite breeding lines. Theor Appl Genet 92:191203

Thomson MJ, Tai TH, McClung AM, Lai XH, Hinga ME, Lobos KB, Xu Y, Martinez CP, McCouch SR (2003)Mapping quantitative trait loci for yield, yield components and morphological traits in an advanced backcross population between Oryza rufipogon and the Oryza sativa cultivar Jefferson. Theor Appl Genet 107:479-493

Tian F, Li DJ, Fu Q, Zhu ZF, Fu YC, Wang XK, Sun CQ (2006)Construction of introgression lines carrying wild rice (Oryza rufipogonGriff.) segments in cultivated rice (Oryza sativa L.) backgroundand characterization of introgressed segments associatedwith yield-related traits. Theor Appl Genet 112:570-580

Wan XY, Wan JM, Weng JF, Jiang L, Bi JC, Wang CM, Zhai HQ (2005)Stability of QTLs for rice grain dimension and endosperm chalkinesscharacteristics across eight environments. Theor Appl Genet 110:1334-1346

Wang SK, Li S, Liu Q, Wu K, Zhang JQ, Wang SS, Wang Y, ChenXB, Zhang Y, Gao CX, Wang F, Huang HX, Fu XD (2015a)The OSSPL16-GW7 regulatory module determines grain shapeand simultaneously improves rice yield and grain quality. NatGenet 47(8):949-954

Wang SK, Wu K, Yuan QB, Liu XY, Liu ZB, Lin XY, Zeng RZ,Zhu HT, Dong GJ, Qian Q, Zhang GQ, Fu XD (2012) Controlof grain size, shape and quality by OSSPL 16 in rice. Nat Genet44(8):950-954

Wang YX, Xiong GS, Hu J, Jiang L, Yu H, Xu J, Fang YX, Zeng LJ, Xu EB, Xu J, Ye WJ, Meng XB, Liu RF, Chen $H Q$, Jing YH, Wang YH, Zhu XD, Li JY, Qian Q (2015b)Copy number variation at the GL7locus contributes to grain size diversity in rice. NatGenet 47(8):944-948

Wang ZY, Second G, Tanksley SD (1992) Polymorphism andphylogenetic relationships among species in the genus Oryza as determined by analysis of nuclear RFLPs. Theor Appl Genet 83:565-581

Xiao JH, Li JM, Grandillo S, Ahn SN, Yuan LP, Tanksley SD, McCouch SR (1998)Identification of traitimproving quantitative trait loci alleles from a wild rice relative, Oryza rufipogon. Genetics 150:899-909

Xie X, Song MH, Jin F, Ahn SN, Suh JP, Hwang HG, Kim YG, McCouch SR(2008)Fine mapping of a yieldenhancing QTL cluster associated with transgressivevariation in an Oryza sativa× O. rufipogon cross. Theor Appl Genet116:613-622 
Xie XB, Song MH, Jin FX, Ahn SN, Suh JP, Hwang HG, McCouch SR (2006)Fine mapping of a grain weight quantitative trait locus on ricechromosome 8 using near-isogenic lines derived from a crossbetween Oryza sativa and Oryza rufipogon. Theor Appl Genet 113:885-894

Xiong YZ, Zhang QF (2010)Genetic and molecular bases of rice yield.Annu Rev Plant Biol 61:421-442

Xu F, Fang J, Ou SJ, Gao SP, Zhang FX, Du L, Xiao YH, Wang HR, SunXH, Chu JF, Wang GD, Chu

CC (2015) Variations in CYP78A13coding region influence grain size and yield in rice. Plant

Cell \&Environ38(4):800-811

Xue P, Zhang YX, Lou XY, Zhu AK, Chen YY, Sun B, Yu P, Cheng SH, Cao LY, Zhan XD (2019) Mapping and genetic validation of a grain size QTL qGS7. 1 in rice(Oryza sativa L.). J Integr Agric 18(8):1838-1850

Yoon DB, Kang KH, Kim HJ, Ju HG, Kwon SJ, Suh JP, Jeong OY, Ahn SN (2006)Mapping quantitative trait loci for yield components and morphologicaltraits in an advanced backcross population between Oryza grandiglumisand the 0 . sativa japonica cultivar Hwaseongbyeo. Theor Appl Genet 112:10521062

Yu JP, Miao JL, Zhang ZY, Xiong HY, Zhu XY, Sun XM, Pan YH, Liang YT, Zhang Q, Rehman RMA, Li JJ, Zhang $\mathrm{HL}$, Li ZC (2018) Alternative splicing of OsLG3b controls grain length and yield in japonica rice.

Plant Biotechnol J 16(9):1667-1678

Yun YT, Chung CT, Lee YJ, Na HJ, Lee JC, Lee SG, Lee KW, Yoon YH, Kang JW, Lee HS, Lee JY, Ahn SN (2016)QTL mapping

of grain quality traits using introgression lines carrying Oryzarufipogonchromosome segments injaponicarice. Rice 9:62

Zhang Q, Yao GX, Hu GL, Chen C, Tang B, Zhang HL, Li ZC (2012)Fine Mappingof qTGW3-1, a QTL for 1000Grain Weight on Chromosome3 in Rice. J Integr Agri 11(6):879-887

Zhang XJ, Wang JF, Huang J, Lan HX, Wang CL, Yin CF, Wu YY, Tang HJ, Qian Q, Li JY, Zhang HS (2012) Rare allele of $O S P P K L 1$ associated with grain length causes extra-large grainand a significant yield increase in rice. Proc Natl Acad Sci USA109:21534-21539

Zhao DS, Li QF, Zhang CQ, Zhang C, Yang QQ, Pan LX, Ren XY, Lu J, Gu MH, Liu QQ (2018) GS9 acts as a transcriptionalactivator to regulate rice grain shape and appearance quality. NatCommun 9(1):1240

Zuo JR, Li

JY (2014)Molecular genetic dissectionof quantitative trait loci regulating rice grain size. Ann Rev Genet 48:99118

\section{Figures}




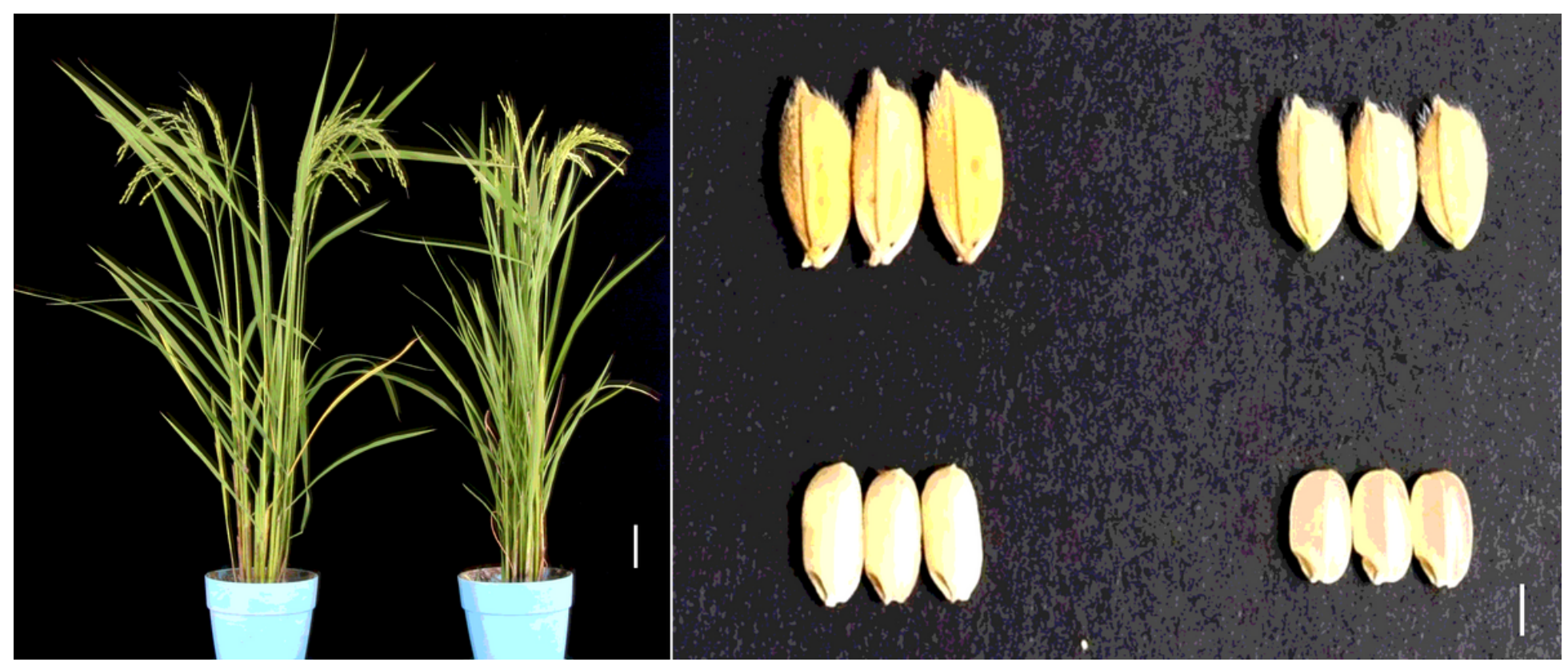

Figure 1

Comparison of whole-plant rice and grain performance between IL188 and Nipponbare. A, Plant type of IL188 (left) and Nipponbare (right). B, Paddy and brown rice grains of IL188 (left) and Nipponbare (right). Bars: 10 $\mathrm{cm}$ in $\mathrm{A}, 6 \mathrm{~mm}$ in $\mathrm{B}$. 

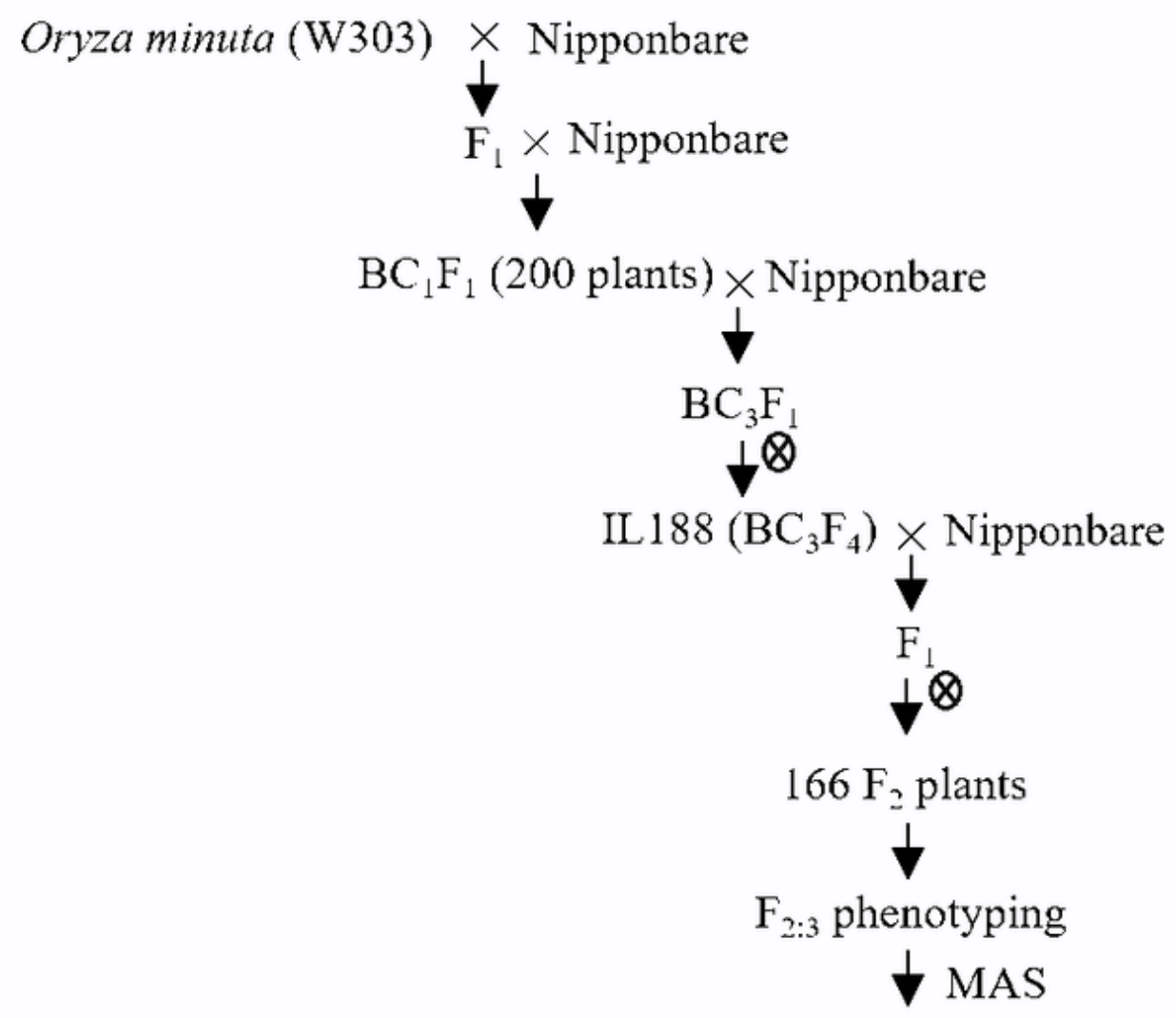

Four plants heterozygous in RM1135-RM11, RM11-Y7-2, RM11-Y7-2, or RM11-Y7-4 $\perp \otimes$

R1 (180 plants), R2 (184 plants), R3 (184 plants), R4 (195 plants)

$\downarrow$ MAS

Four plants heterozygous in Y7-4-RM21787, Y7-4-

RM455, RM21787-Y7-12, or RM21787-Y7-13

$\perp \otimes$

R5 (130 plants), R6 (144 plants), R7 (146 plants), R8 (140 plants)

\section{Figure 2}

A scheme showing how plants materials were developed. 

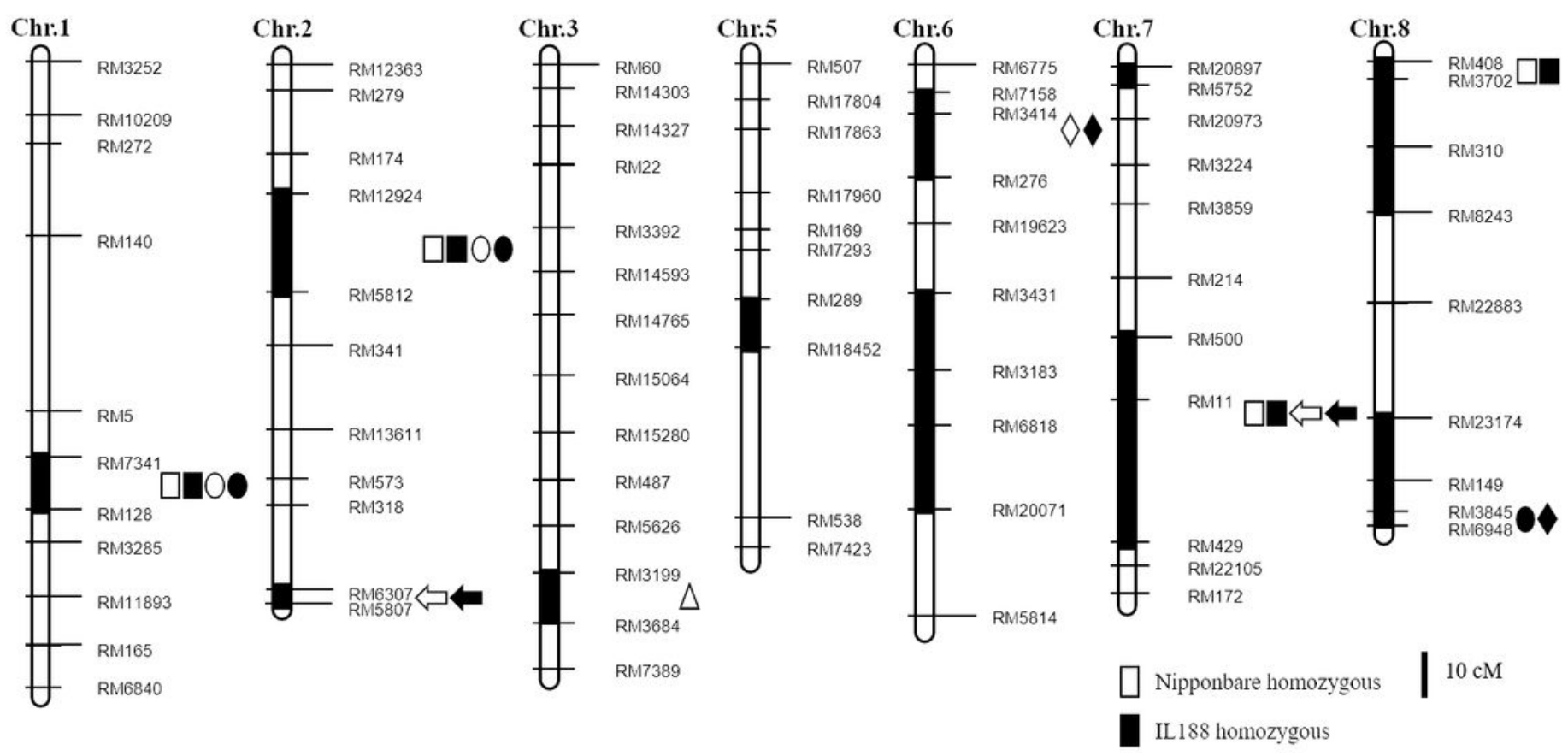

$\mathrm{F}_{2} \mathrm{GL} \Delta \mathrm{F}_{2} \mathrm{GW} \mathrm{OF}_{\mathrm{F}_{2} \mathrm{LWR}} \diamond_{\mathrm{F}_{2} \mathrm{GT}}\left\langle\mathrm{F}_{2} \mathrm{TGW}\right.$

$\mathrm{F}_{2: 3} \mathrm{GL} \backslash \mathrm{F}_{2: 3} \mathrm{GW} \mathrm{F}_{2: 3} \mathrm{LWR} \mathrm{F}_{2: 3} \mathrm{GT} \sim \mathrm{F}_{2: 3} \mathrm{TGW}$

\section{Figure 3}

Genetic linkage map showing QTL positions detected in the F2 and F2:3 populations. White and black shapes indicate $\mathrm{F} 2$ and $\mathrm{F} 2: 3$ population, respectively.
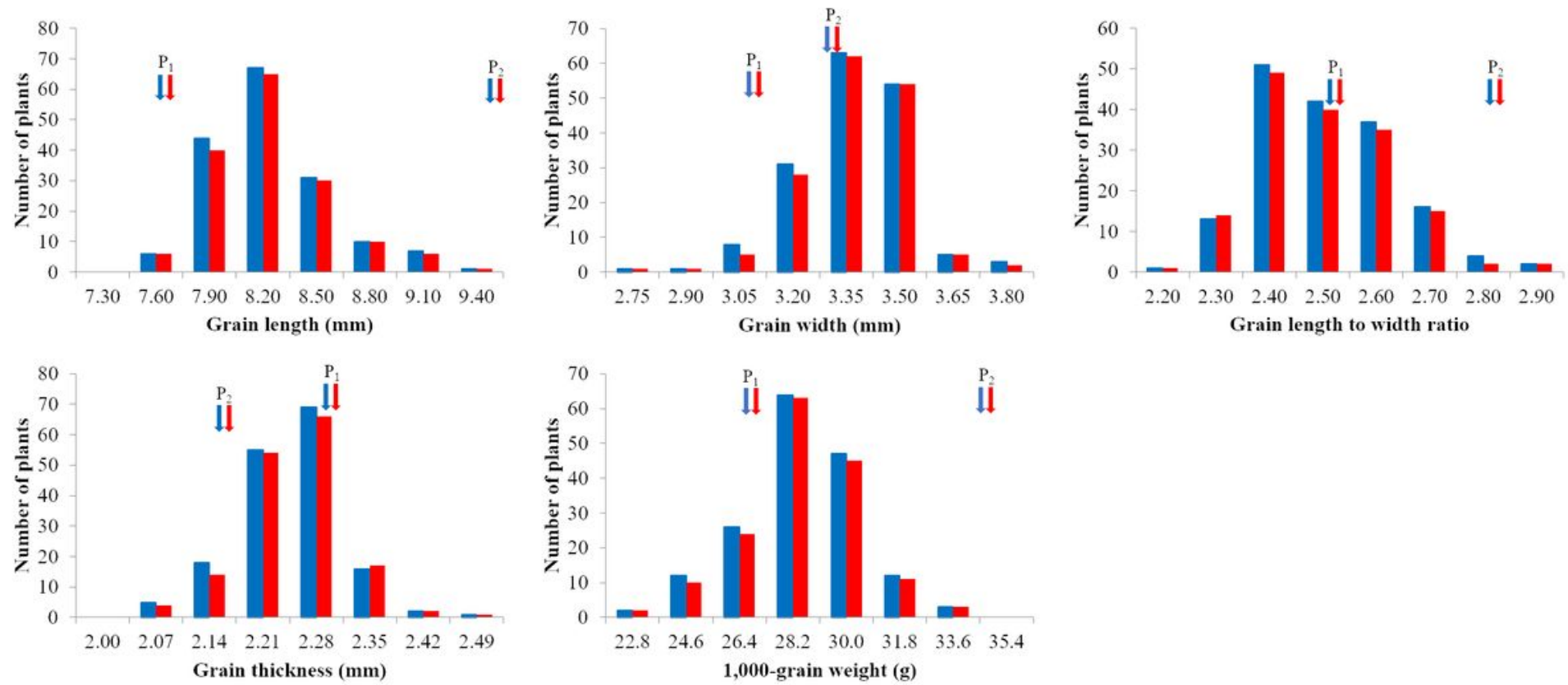

Figure 4

Frequency distribution of five grain shape traits in the F2 and F2:3 populations. (P1: Nipponbare, P2: IL188). The vertical axis of each figure represents the number of $\mathrm{F} 2$ and F2:3 plants, blue bars and red bars indicate $\mathrm{F} 2$ and $\mathrm{F} 2: 3$, respectively. 


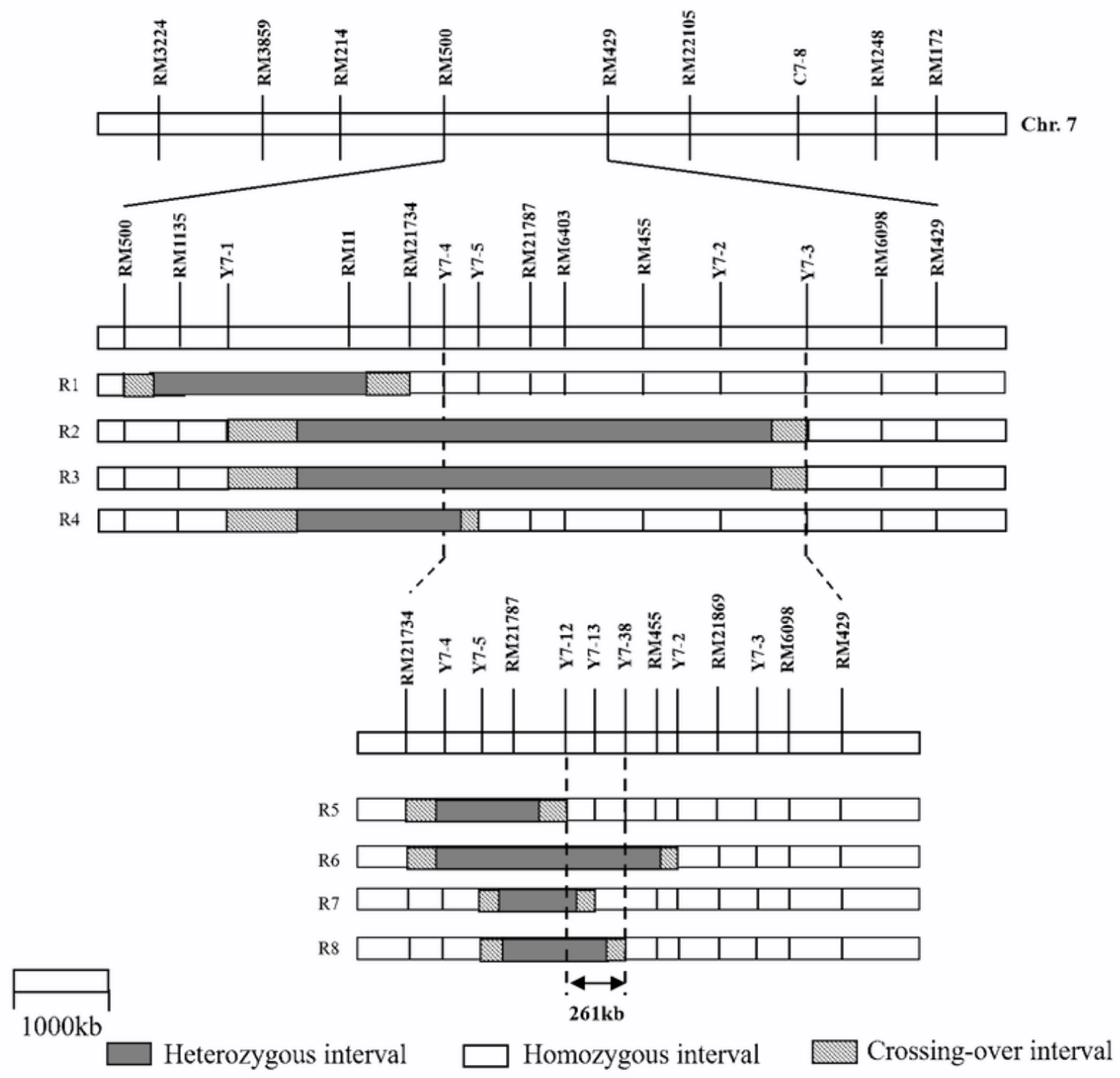

Figure 5

Genotypes compositions of NIL populations in the target region. NIL, near-isogenic line. 


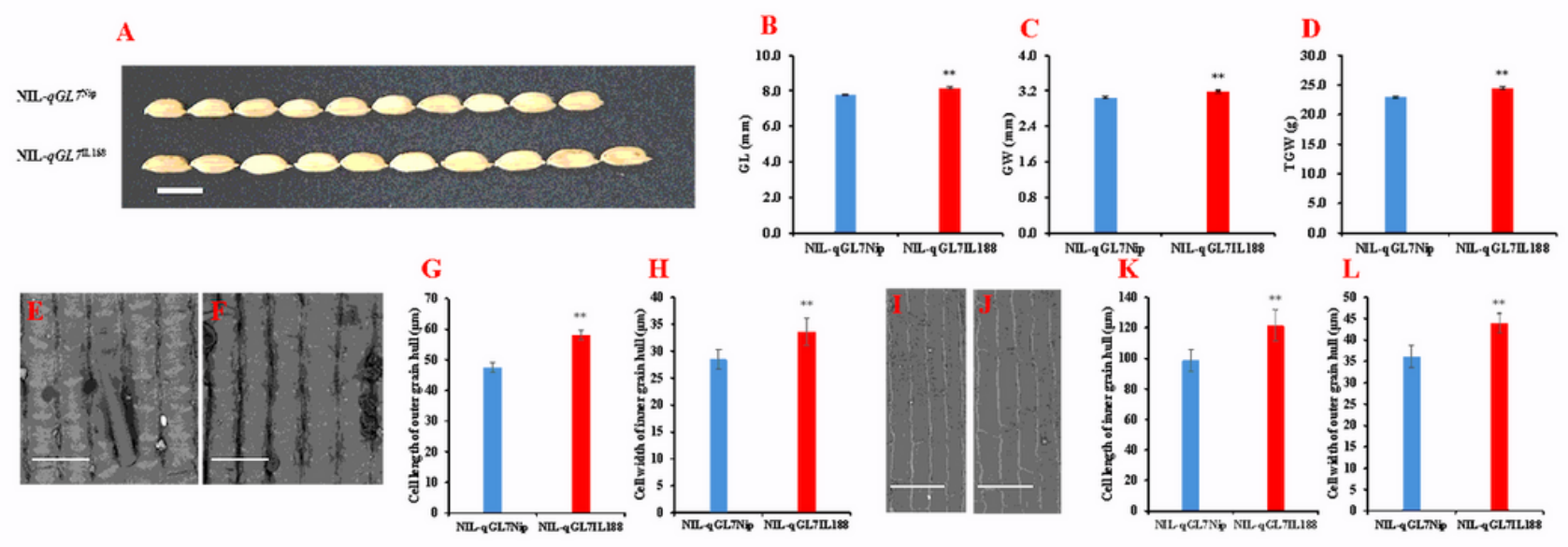

Figure 6

Comparison of grain size and spikelets epidermal cells between NIL-qGL7Nip and NIL-qGL7IL188. A, Mature grains of NIL-qGL7Nip and NIL-qGL7IL188. Scale bar, $6 \mathrm{~mm}$. B-D, Grain length (GL), grain width (GW), and 1000-grain weight (TGW) for NIL-qGL7Nip and NIL-qGL7IL188. Data are given as mean $\pm(n=20)$. ** indicate significant difference at 0.01 level. E-F, Outer epidermal cells of grain hulls of NIL-qGL7Nip and NIL-qGL7IL188. Bars $=100 \mu \mathrm{m} . \mathrm{G}-\mathrm{H}$, The average length and width of outer epidermal cells. $(n=10) . I-J$, Inner epidermal cells of grain hulls of NIL-qGL7Nip and NIL-qGL7IL188. Bars $=100 \mu \mathrm{m}$. K-L, The average length and width of inner epidermal cells. $(n=10)$.

\section{Supplementary Files}

This is a list of supplementary files associated with this preprint. Click to download.

- Supplementarytable.docx

- SupplementaryFig.1.tif 